\title{
Normal variance in renal size in relation to body habitus
}

\author{
Werner S Harmse, MB ChB \\ Department of Diagnostic Radiology, Faculty of Health Sciences, University of the Free State, Bloemfontein
}

Corresponding author: W Harmse (wsharmse@mweb.co.za)

\begin{abstract}
Objective. Renal length determination is common in everyday radiology practice. However, a normal range of kidney sizes may not apply to people of all body habitus. This study investigates this relationship in order to determine normal ranges in relation to body habitus. A secondary aim was to evaluate the relationship of renal size to gender and race.

Methods. Kidney lengths were measured on oblique coronal reformatted CT images of 514 patients who received routine abdominal CT scans for conditions unrelated to renal pathology. The patients had normal serum creatinine levels, no history of renal disease, no renal masses, and normal-appearing kidneys on CT. Weight, height, race and gender of the patients were recorded.

Results. The mean renal length was $108 \mathrm{~mm}$ with a standard deviation of $9.82 \mathrm{~mm}$. Statistical analysis demonstrated a relationship between kidney size and body weight and height, both individually and collectively. The most accurate prediction model was 'kidney size $=49.18+0.21 \times$ weight $+0.27 \times$ height', with a R2-value of 0.32 . Additionally, kidneys were generally larger in the white population than in the black, and also in males than females.

Conclusion. Normal renal size varies according to patients' body habitus. This variation can be expressed as a function of body weight and height, which can be represented by a nomogram and used as an easy reference in clinical practice.
\end{abstract}

Renal size is an important parameter for clinical assessment of patients with diabetes, renal artery stenosis or chronic renal failure, and for assessment of kidney transplant candidates. Renal sizes facilitate differentiation between chronic and acute renal failure, and when a decision has to be made on whether to take renal biopsies or not.

It is therefore imperative to have accurate data regarding normal renal sizes. Numerous studies have established normal renal lengths for the average adult population, which is approximately $11 \mathrm{~cm} \pm 1$ $\mathrm{cm},{ }^{1-3}$ with only slight variation among different authors. However, limited research has been done on the variation of normal renal size in relationship to body habitus, as well as gender and race. In a study performed in 1991 with a volunteer Danish population in Copenhagen, ${ }^{4}$ a definite association in adults between renal size and body habitus was found. However, no specific model was developed, as this was not the primary aim of the investigation. This study was also on a single racial group. ${ }^{4}$ Fernandes et al. ${ }^{5}$ found differences in measurements between different population groups in a study performed in Brazil. Most previous studies also used sonography or intravenous urography to measure renal dimensions. In 2007, Kang et al. ${ }^{6}$ evaluated different radiological methods of estimating renal size, and concluded that coronal CT scans were the most accurate radiological method for doing so. A clinical dilemma facing radiologists and clinicians may arise in deciding whether larger kidney sizes may be acceptable for a big patient or, conversely, if a small kidney can be accepted as normal for a smaller adult patient. The aim of this study was (i) to evaluate the relationship between renal length and different body habitus indices, including weight, height, body mass index (BMI) and body surface area (BSA) by using CT images; (ii) to determine whether a relationship exists and, if so, to establish a normal range for renal lengths in relation to body habitus; and (iii) to establish a possible relationship between renal size and age, gender and/or race.

Ethical approval to conduct the investigation was granted by the Ethics Committee of the Faculty of Health Sciences, University of the Free State.

\section{Methods}

An analytical cross-sectional study was performed on patients attending the diagnostic radiology department at the National District Hospital, Bloemfontein. All patients receiving routine CT scans of the abdomen from July 2009 to June 2010 were evaluated for inclusion in the study. These patients were scanned on a GE (General Electric) 8-slice Brightspeed CT scanner at a section thickness of $2.5 \mathrm{~mm}$.

Patients were limited to adults between 18 and 70 years of age. The patients were from central South Africa and represented different racial groups, including black, white, coloured and Asian.

Patients with diseases or pathology that might influence renal size were excluded from the study. Exclusion criteria applied to the patients on arrival were (i) known chronic renal disease, (ii) previous renal surgery, (iii) patient too ill to allow weighing and measuring, and (iv) elevated serum creatinine $>100 \mu \mathrm{mol} / \mathrm{l}$ (upper limit of normal at the local laboratory).

Patients included in the study were weighed and measured, and their age, gender and race recorded. After receiving their scans, their images were evaluated and a further 4 exclusion criteria were applied, namely (i) incidental finding of renal masses on CT scan, including a solitary cyst $>4 \mathrm{~cm}$, multiple cysts $(>4)$, polycystic renal disease, ${ }^{4}$ (ii) hydronephrosis, (iii) a single kidney, and (iv) congenital abnormalities including ectopic and fused kidneys.

Both the left and right kidneys of the remaining patients were measured, which was done by using multiplanar reformations on Philips iSite Advanced Visualisation Software. Oblique coronal planes were used to measure the maximum diameter of the kidneys. Standard coronal reformatted images were individually tilted along the longitudinal axes for both left and right kidneys, using a reference line on sagittal images. 


\section{ORIGINAL ARTICLE}

The maximum length of the kidneys was then measured on these oblique coronal images (see Fig. 1).

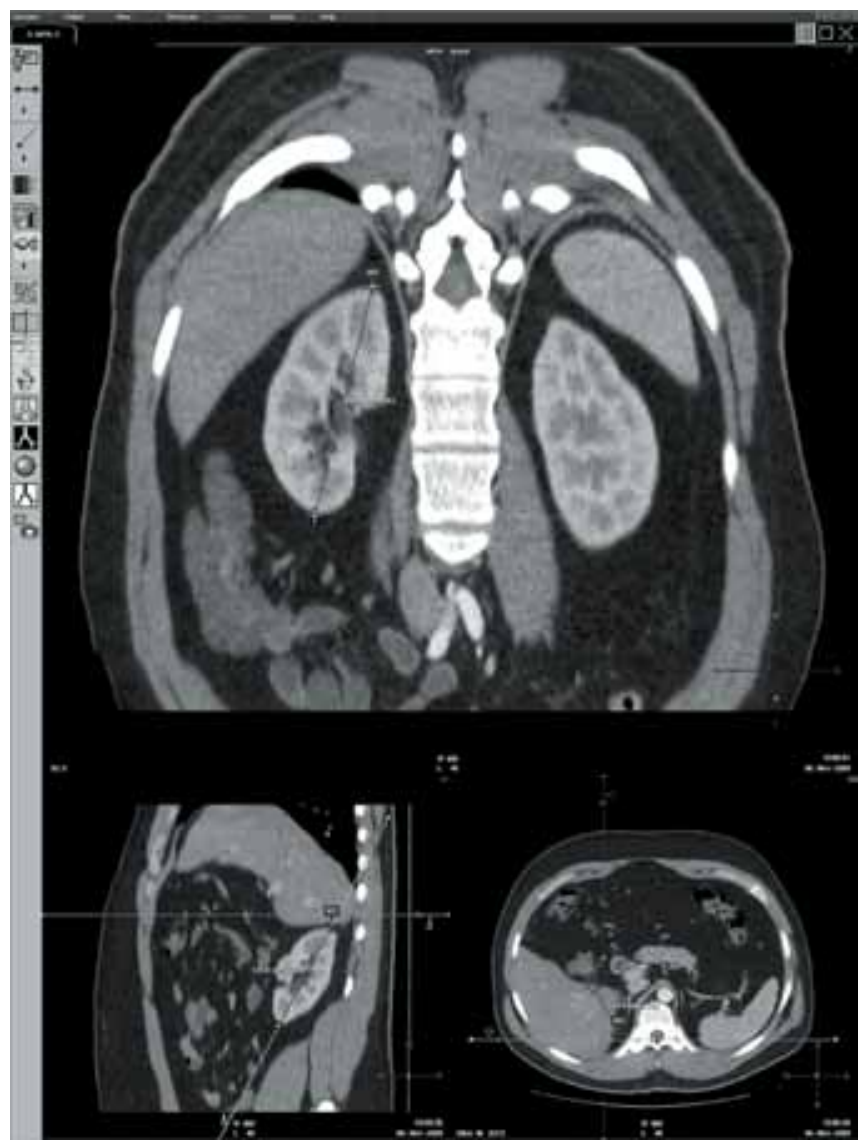

Fig. 1. Oblique coronal images used to measure maximum renal length.

\section{Results}

A total of 677 patients from July 2009 to June 2010 with no known renal disease, no previous renal surgery and a serum creatinine level $<100 \mu \mathrm{mol} / \mathrm{l}$ received abdominal CT scans at National Hospital in Bloemfontein. One hundred and sixty-three of these patients were excluded owing to hydronephrosis, visible renal atrophy, renal masses, multiple cysts or congenital renal variants, giving a remainder of 514 patients who were included in the study.

On average, the left kidney was $2.06 \mathrm{~mm}(p<0.001)$ larger than the right kidney, with differences ranging from $23 \mathrm{~mm}$ larger to $17 \mathrm{~mm}$ smaller. For each patient, the mean kidney size between left and right was determined and used for further analysis, being referred to as 'kidney size' unless stated otherwise. Kidney sizes varied from $80 \mathrm{~mm}$ to $134 \mathrm{~mm}$, with a mean size of $108 \mathrm{~mm}$. As illustrated in Fig. 2, the kidney sizes showed a symmetric distribution with a standard deviation of $9.82 \mathrm{~mm}$

Firstly the relationship between kidney size and weight was explored. The regression model (see Fig. 3) was indicative of a significant relationship between kidney size and weight, with a unit increase in the patient's weight being associated with an increase of 0.2562 units in kidney size. The $\mathrm{R}^{2}$ of the regression model is 0.2464 , indicating that weight can explain $24.64 \%$ of the variation in kidney size.

The relationship between kidney size and the patient's height is shown in Fig. 4 with the estimates of a regression model. The regression model indicates a significant relationship between kidney size and

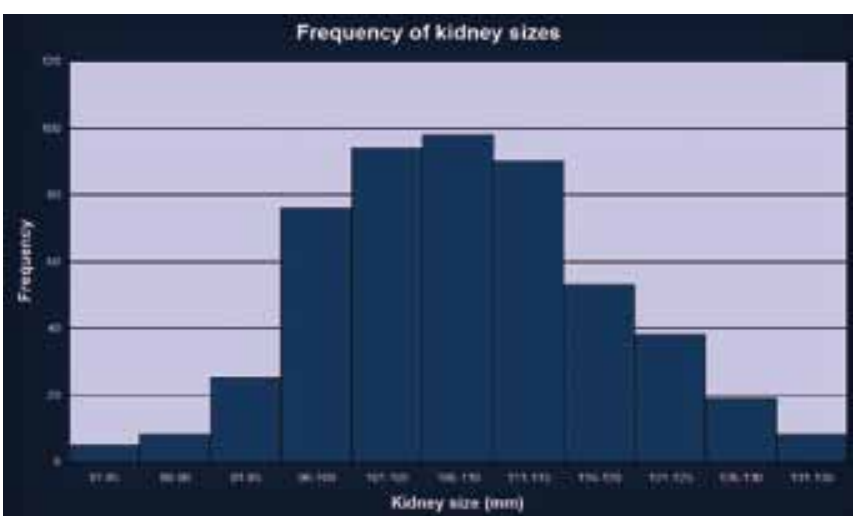

Fig. 2. Distribution of kidney sizes.

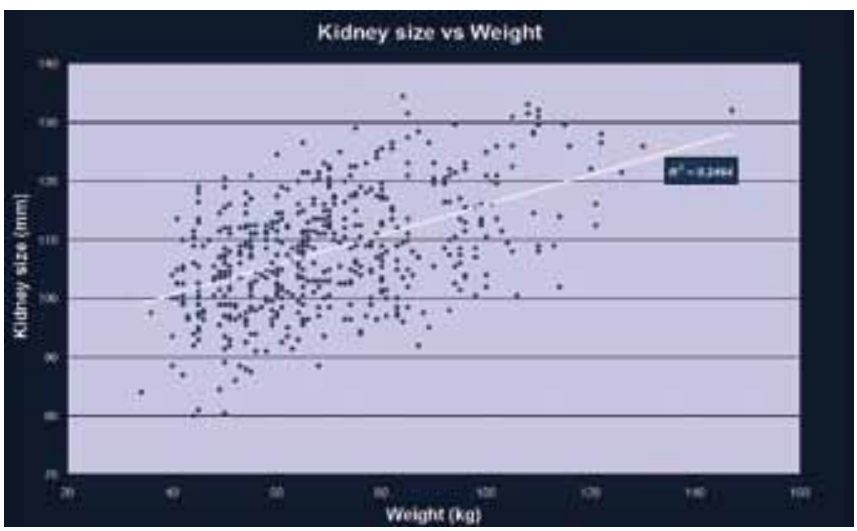

Fig. 3. Kidney size in relation to body weight.

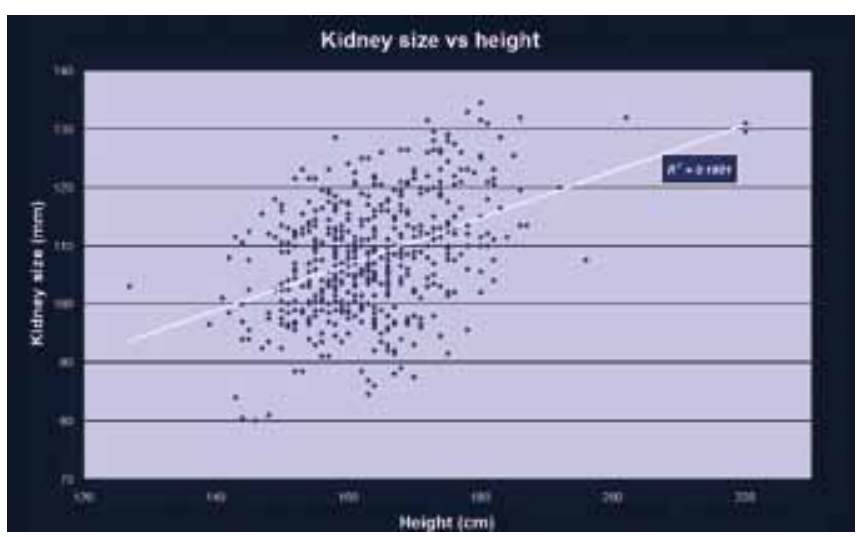

Fig. 4. Kidney size in relation to body height.

length, with a unit increase in length being associated with an increase of 0.39868 units in kidney size. The $\mathrm{R}^{2}$ of the model is 0.1801 .

Fig. 5 illustrates the relationship between kidney size and the patient's body mass index (BMI = weight in $\mathrm{kg}$ divided by height in meters squared) with the estimates of a linear regression model. BMI is significant in the regression model, with one unit increase in BMI being associated with an increase in kidney size of 0.42180 units. However, the $\mathrm{R}^{2}$ of the model is only 0.08708 , indicating that BMI can explain only $8.7 \%$ of the variation in kidney sizes.

The relationship between kidney size and the patient's body surface area (BSA) is shown in Fig. 6 with the estimates of a linear regression model. BSA was calculated using the Mosteller formula, ${ }^{7}$ being BSA $\left(\mathrm{m}^{2}\right)=([\text { height }(\mathrm{cm}) \mathrm{x} \text { weight }(\mathrm{kg})] / 3600)^{1 / 2}$. BSA is significant in the regression model, with one unit increase in BSA being associated with an increase in kidney size of 20.359 units. The $\mathrm{R}^{2}$ of the model is 0.2975 . 


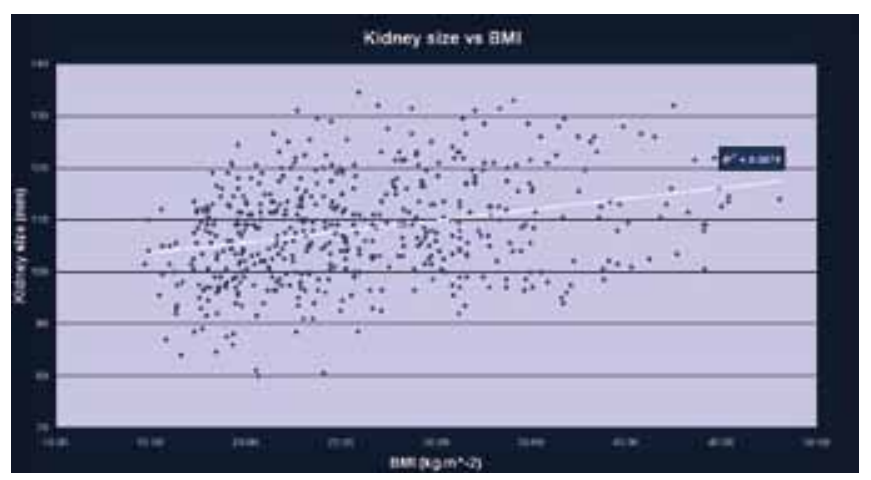

Fig. 5. Kidney size in relation to body mass index.

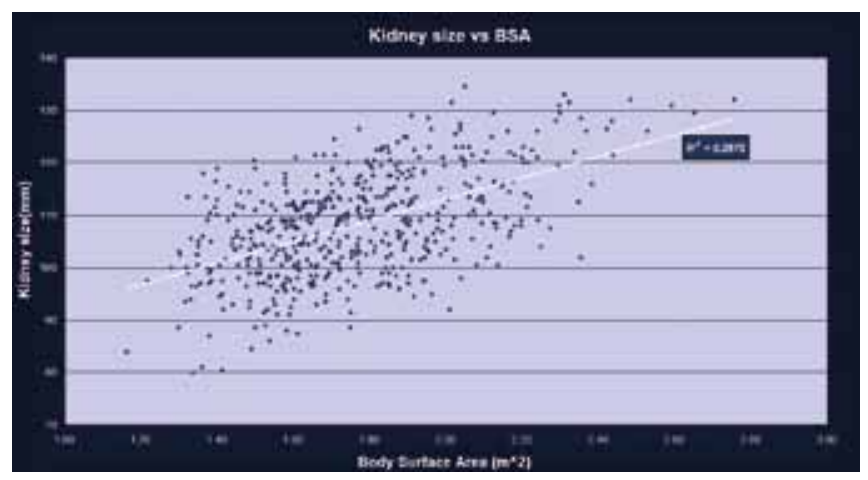

Fig. 6. Kidney size in relation to body surface area.

Regarding these variables (weight, height, BMI and BSA), BSA best explains the variance in kidney size. However, a model was also fitted with both weight and length, and both these parameters were significant in the model. The estimated model is:

Kidney size $(\mathrm{mm})=49.18109+0.20605 \mathrm{x}$ weight $(\mathrm{kg})+0.27360 \mathrm{x}$ height $(\mathrm{cm})($ standard error $=8.1 \mathrm{~mm})$

When comparing this model with the model using only BSA, we see that 'weight + height' has an adjusted $\mathrm{R}^{2}$ of 0.3191 , while the model with only BSA has an adjusted $\mathrm{R}^{2}$ of 0.2962 . This difference indicates that 'weight + length' is a better model than using only BSA. For further discussion, ' $0.20605 \mathrm{x}$ weight $(\mathrm{kg})+0.27360 \mathrm{x}$ height $(\mathrm{cm})$ ' is referred to as the 'body habitus constant.

Further analyses of data were done regarding differences in renal size related to age, gender and race. No clear relationship between age and kidney size was found (see Fig. 7). Table I illustrates the gender and racial distribution, with the average renal sizes, as well as body habitus constants for each group.

Owing to the coloured and Asian groups being too small to make a meaningful contribution to the findings, their results were excluded from specific race group analyses. Kidney sizes were generally larger in males than females $(3.2 \mathrm{~mm}, p<0.05)$ and also in whites than blacks ( 9.1 $\mathrm{mm}, p<0.05)$. Body habitus may be regarded as a confounding factor in interpreting these results when considering our initial analysis. The mean body habitus constant of males was 61.0, and 57.7 in females, which is similar to the findings on gender-related differences in renal sizes. This finding suggests that the difference in renal sizes between gender groups is most likely due to the difference in body habitus between male and female, rather than a true difference due to gender. When correcting for this difference in body habitus, a much smaller difference in renal sizes of only $0.1 \mathrm{~mm}(p=0.6)$ is found between male and female patients, which is not clinically significant.

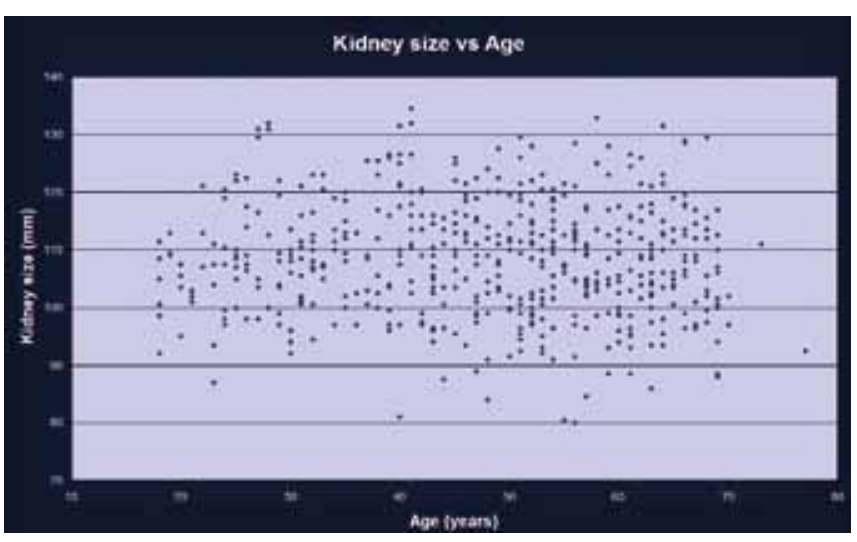

Fig. 7. Kidney size in relation to age.

Similar results were found comparing the renal sizes and body habitus constants of blacks and whites. Blacks had on average smaller renal sizes $(9.1 \mathrm{~mm})$, but also smaller body habitus constants than whites. This finding therefore suggests that the difference in renal sizes found between different racial groups may also be due to the difference in body habitus, rather than a true racial difference. When correcting renal sizes for the differences in body habitus, a difference of only $3.2 \mathrm{~mm}(p$ $<0.05)$ is observed, which is substantially smaller than the $9.1 \mathrm{~mm}$ and, although statistically significant, unlikely to be clinically significant. The differences in body habitus may be attributed to a number of different genetic, cultural and socio-economic factors.

\section{Discussion}

Kidney sizes are important in the evaluation of renal disease for both radiologists and clinicians. Coronal reformatted CT scans were used for measurement purposes as this approach was previously proven to be the most accurate way of measuring kidney sizes on imaging studies. ${ }^{6}$ Furthermore, CT scans are more reproducible and less operatorand patient-dependent than ultrasound. In clinical practice, however, ultrasound is more readily available and free of radiation, and will most likely remain the preferred method for evaluating renal sizes. The mean renal size is $110 \mathrm{~mm}( \pm 10 \mathrm{~mm}),{ }^{1-3}$ with only minimal variation reported by different authors. In our study population, mean renal sizes were fairly similar, with a mean size of $108.2 \mathrm{~mm}$ and a standard deviation of $9.82 \mathrm{~mm}$. A clinical dilemma might arise in patients with a kidney size bigger or smaller than this; one then needs to decide whether this can be accepted as normal for the particular patient owing to his/her

Table I. Mean renal size and body habitus constant related to gender and race.

\begin{tabular}{llll}
\hline & $\begin{array}{l}\text { Number of } \\
\text { participants }\end{array}$ & $\begin{array}{l}\text { Mean renal } \\
\text { size }(\mathbf{m m})\end{array}$ & $\begin{array}{l}\text { Body habitus } \\
\text { constant }\end{array}$ \\
\hline $\begin{array}{l}\text { Total } \\
\text { Gender }\end{array}$ & 514 & 108.2 & 59.01 \\
Female & 306 & 106.9 & 57.7 \\
Male & 208 & 110.1 & 61.0 \\
Race & & & \\
African & 342 & 105.5 & 57.4 \\
Caucasian & 146 & 114.6 & 63.2 \\
Coloured & 25 & 108 & 56.5 \\
Asian & 1 & 107.5 & 58.8 \\
\hline
\end{tabular}




\section{ORIGINAL ARTICLE}

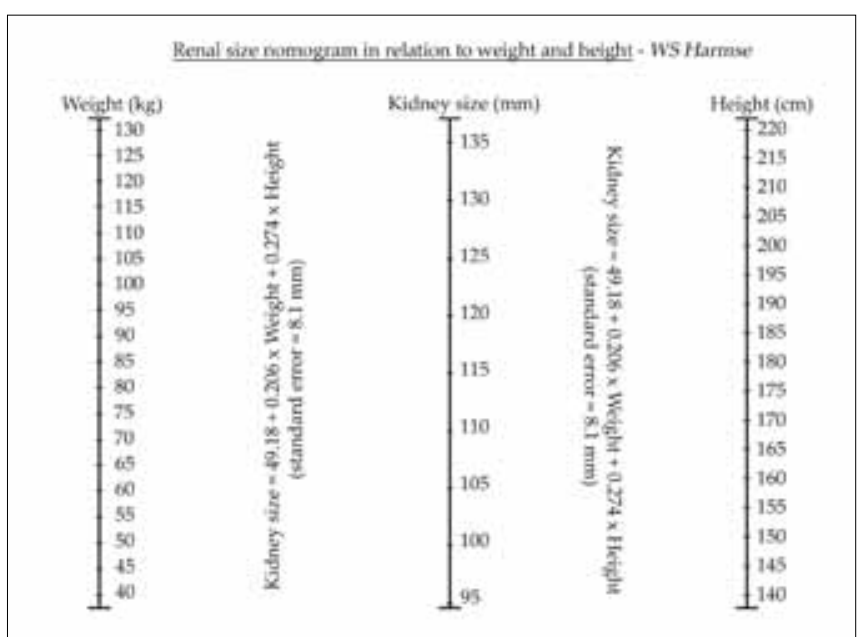

Fig. 8. Renal size nomogram in relation to weight and height.

body habitus. As expected, I found a relationship between renal sizes and body habitus, which supports the findings of Emamian et al., ${ }^{4}$ who demonstrated a correlation between renal sizes and BSA and height. From a physiological perspective, this finding would make sense, as patients with a bigger body habitus will have a larger blood volume requiring larger kidneys for filtration. Glodny et al. ${ }^{8}$ also demonstrated a relationship between body height and renal length. They, however, found a strong relationship between renal length and BMI, which differed from my results where the relationship with BMI was the weakest of all the factors studied. They also did not study the influence of body weight, BSA or other combinations of weight and height. ${ }^{8}$

The most accurate model proposed by the author for predicting renal sizes is 'Kidney size $(\mathrm{mm})=49.18109+0.20605 \mathrm{x}$ weight $(\mathrm{kg})+0.27360 \mathrm{x}$ height $(\mathrm{cm})$. This model had a standard error of $8.1 \mathrm{~mm}$. It is a cumbersome formula to use in daily practice, and I consequently constructed a nomogram (Fig. 8) to allow easy reference in the clinical situation.

When a patient's weight and height is known, a straight line can be drawn between these values on the scales representing weight (left) and height (right). This line will intersect the central scale indicating the approximate kidney size expected for this patient.

No relationship between age and renal size was found, although variation between different genders and races was observed. The variation in kidney sizes, however, was similar to the differences in body habitus, and more likely due to body habitus rather than inherent differences. It could further be argued that the differences in body habitus are in all probability the result of numerous socio-economic and cultural factors.

A few limitations were experienced during this study and need to be noted. The measurements were made by a single observer, which was mostly because of lack of personnel. Reformations were also done on $2.5 \mathrm{~mm}$ slices which were the limit on routine abdominal scans on the available equipment. These factors limited the accuracy of the results, leading to a larger variation of normal values. The standard error of estimation of our model was $8.1 \mathrm{~mm}$. By increasing sample size and improving the accuracy of the measurements, it should be possible to decrease this error. Nevertheless, the results were regarded as valid, especially since normal renal sizes do have a range of values spanning approximately $20 \mathrm{~mm}$ in variation, rather than a single specific value.

\section{Conclusion}

Normal renal sizes vary according to patients' body habitus, and can be expressed as a function of body weight and height. This function can be represented by a nomogram that can be used as an easy reference in clinical practice. No relationship was found between renal sizes and age. Variations were found in renal sizes of different genders and races, although these appeared to be related to differences in body habitus and not true inherent differences.

Acknowledgements. Professor Gina Joubert, Department of Biostatistics, University of the Free State, and Christiaan Marais are thanked for the statistical analysis of data, and Daleen Struwig, medical writer, Faculty of Health Sciences, University of the Free State, for technical and editorial preparation of the manuscript for publication.

1. Brandt TD, Neiman HL, Dragowski MJ, Bulawa W, Claycamp G. Ultrasound assessment of normal renal dimensions. J Ultrasound Med 1982;1:49-52.

2. Kabala J, Roobottom C. The urogenital tract: anatomy and investgations. In: Sutton D, ed. Textbook of Diagnostic Radiology and Imaging. 7th ed. London: Churchill Livingstone, 2003:828-885.

3. Dahnert W, ed. Radiology Review Manual. 6th ed. Philadelphia: Lippincott Williams \& Wilkins, 2007.

4. Emamian SA, Nielsen MB, Pedersen JF, Ytte L. Kidney dimensions at sonography: correlation with age, sex, and habitus in 665 adult volunteers. Am J Roentgenol 1993;160:83-86.

5. Fernandes MMR, Lemos CCS, Lopes GS, et al. Normal renal dimensions in a specific population. Int Braz J Urol 2002;28:510-515.

6. Kang K, Lee YJ, Park SC, et al. A comparative study of methods of estimating kidney length in kidney transplantation donors. Nephrol Dial Transplant 2007;22:2322-2327.

7. Mosteller RD. Simplified calculation of body-surface area (letter). N Engl J Med 1987;317:1098

8. Glodny B, Unterholzner V, Taferner B, et al. Normal kidney size and its influencing factors - a 64-sliceMDCT study of 1.040 asymptomatic patients. BMC Urology 2009;9:19-31. 